\title{
Is Bony Knee Alignment Representative of the True Joint Surface in Skeletally Immature Patients? A Magnetic Resonance Imaging Study
}

\author{
Stephen D Bigach ${ }^{1}$, Christopher N Carender ${ }^{2}$, Raymond W Liu ${ }^{3}$
}

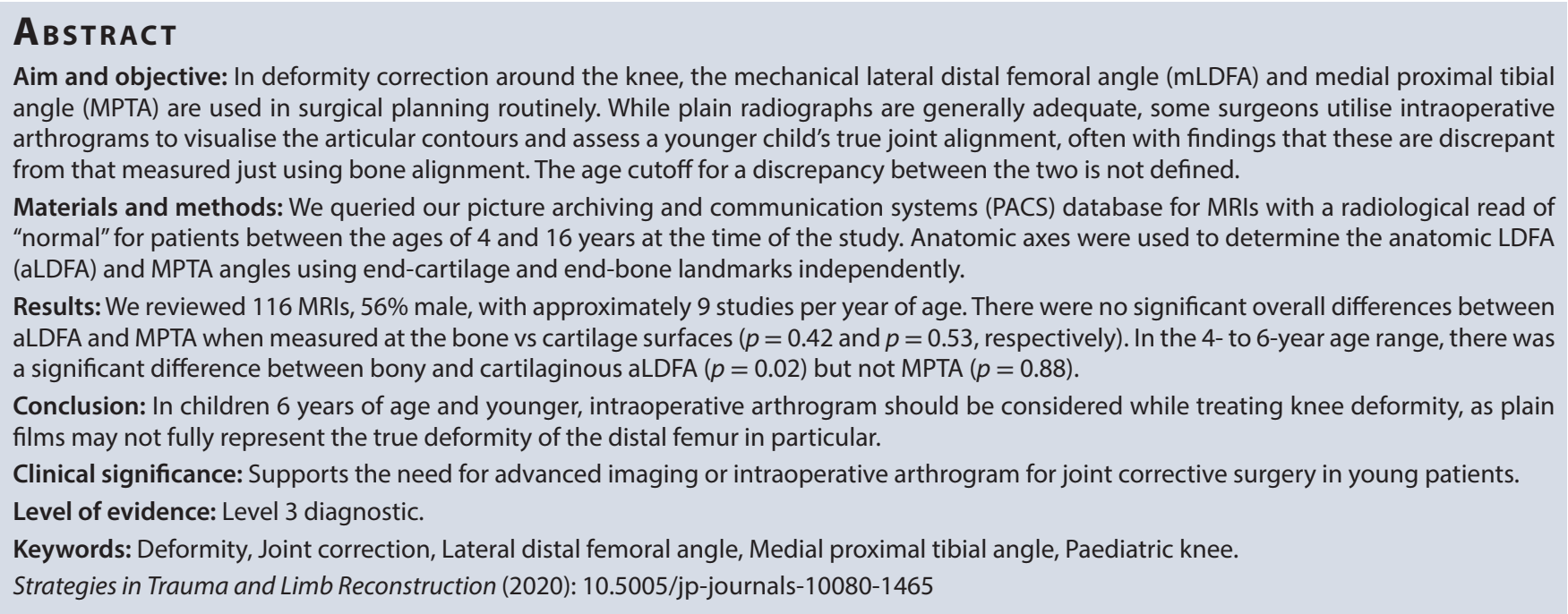

\section{INTRODUCTION}

In deformity correction around the knee, the mechanical lateral distal femoral angle (mLDFA) and medial proximal tibial angle (MPTA) are used in surgical planning routinely. In the absence of a contralateral normal limb to compare to, surgeons typically rely on normative population values for surgical correction. Normative mLDFA and MPTA values have been defined and corroborated for adults in the literature. ${ }^{1-5}$ At around 18 months of age, the normal knee transitions from varus to valgus angulation. ${ }^{6} \mathrm{~A}$ maximum valgus angulation of the knee occurs around 2-3 years of age before returning to a milder valgus angulation similar to that of the adult. ${ }^{6}$ These anatomical changes must be considered while evaluating young patients with joint deformity. As such, Sabharwal et al. ${ }^{7}$ suggested using age-based normative values while performing limb deformity corrections in children under the age of 7 .

The standard for measuring knee joint deformity is a standing full-length AP radiograph from hip to ankle. These films allow for the measurement of the mechanical and anatomical axes of the lower extremity and calculation of several joint angles and parameters that are crucial to corrective surgery. ${ }^{4,5}$ Measurements utilising the techniques described by Paley ${ }^{1}$ are increasingly difficult in younger patients given the amount of unossified cartilage around the joint. ${ }^{8}$ Surgeons have recognised this challenge and have begun to utilise alternative methods to measure or visualise the joint. A previous article by Ferguson and Fernandes has suggested using the physis as a surrogate joint line on plain radiographs. ${ }^{9}$ Intraoperative
${ }^{1}$ Department of Orthopaedic Surgery, McGaw Medical Center of Northwestern University, Chicago, Illinois, USA

${ }^{2}$ Department of Orthopaedics, University of lowa Hospitals and Clinics, lowa City, lowa, USA

${ }^{3}$ Department of Pediatric Orthopaedics, Rainbow Babies and Children's Hospital at Case Western Reserve University, Cleveland, Ohio, USA

Corresponding Author: Raymond W Liu, Department of Pediatric Orthopaedics, Rainbow Babies and Children's Hospital at Case Western Reserve University, Cleveland, Ohio, USA, Phone: +216-844-7613, e-mail: raymond.liu@uhhospitals.org

How to cite this article: Bigach SD, Carender CN, Liu RW. Is Bony Knee Alignment Representative of the True Joint Surface in Skeletally Immature Patients? A Magnetic Resonance Imaging Study. Strategies Trauma Limb Reconstr 2020;15(2):79-83.

Source of support: Nil

Conflict of interest: None

arthrograms are utilised by some surgeons including the senior author in cases of younger patients but, to our knowledge, there are no definitive studies in the literature to suggest an age group where arthrograms or even preoperative MRIs may add benefit for operative planning as compared to full-length standing AP radiographs. We designed an $\mathrm{MRI}$-based study to evaluate the relationship between age and discrepancy between the measurements taken from the bony and cartilage surfaces (bony vs articular contours) about the knee. 


\section{Materials and Methods}

Institutional Review Board (IRB) approval at our institution was granted for this retrospective chart review study. We queried our picture archiving and communication systems (PACS) database for MRIs of the knee in patients aged 4-16 years obtained between 2007 and 2017. MRIs were excluded if the following were present: the musculoskeletal radiology report indicated any form of internal derangement of the knee; the patient's knee was deemed too flexed at the time of the MRl; the joint cartilage did not articulate on any MRI slice (i.e., discoid meniscus or variant) or if the quality of the T1-weighted coronal image was deemed insufficient for accurate measurement. We sought to include 10 studies for each year of the range of ages 4-16 with a goal of 1:1 male:female ratio. Age groups were filled with the most recent studies first. All measurements used for analysis were made by a single author (SB). Twenty studies, with one or two studies per age group, were selected at random for interobserver and intraobserver reliability studies. Two authors
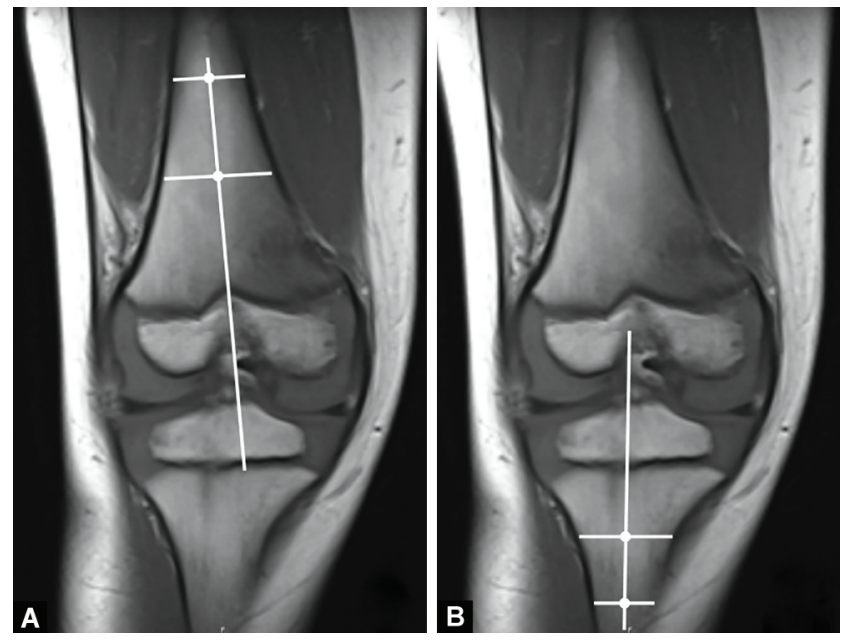

Figs $1 \mathrm{~A}$ and $\mathrm{B}$ : MRI images demonstrating the anatomical axes. The midpoint of two transverse lines proximal to the metaphyseal flare in the distal femur (A), and distal to the metaphyseal flare in the proximal tibia (B), are used to draw anatomic lines
( $\mathrm{SB}$ and $\mathrm{CC}$ ) chose the optimal images and made measurements independently using the same protocol. Interclass correlation coefficients were calculated for each angle measurement, then intraclass correlation coefficients were calculated with the primary measurer performing two independent image selections and measurements over 2 months apart. Following established recommendations by Fleiss, ${ }^{10}$ we considered a coefficient of $<0.4$ to be poor, $0.4-0.75$ to be fair to good and $>0.75$ to be excellent.

\section{Measurements}

Measurements were made on the T1 coronal image of all MRIs. The specific slice of the MRI used for angle measurement was that which demonstrated the most contact between the distal femur (DF) and proximal tibia (PT). In the majority of cases, this cut demonstrated the most distal extent of the DF at both the medial and lateral condyles as well as the most proximal portions of the PT medially and laterally. If this was not the case, the measurements were made using these points super-imposed from other MRI slices. A total of five lines were drawn: the anatomical axis of the femur, the anatomical axis of the tibia, the cartilaginous joint line, the end bone alignment of the DF and the end bone alignment of the PT.

\section{Anatomical Axes}

The anatomical axes of the femur and tibia were drawn using the MRI slice that best demonstrated the anatomy of each respective bone (this was not always the same slice). Two lines were drawn perpendicular to the bone and the anatomical axis was drawn through the midpoint of these lines (Fig. 1). Given the constraints of the MRI image, these lines were drawn at the DF and PT beyond the level of the metaphyseal flare. If the axis was drawn using a separate MRI slice from that which was selected for angle measurement, the line was transposed to the measurement slice.

\section{End Bone, End Cartilage and Joint Lines}

The distal femur end bone line was drawn by connecting the most convex aspect of the ossified medial femoral condyle with the most convex aspect of the lateral femoral condyle (Fig. 2A). The points for the PT were more difficult to precisely define, especially in the younger specimens. To define these points, we drew imaginary lines originating at the distal femoral points that were orthogonal to the
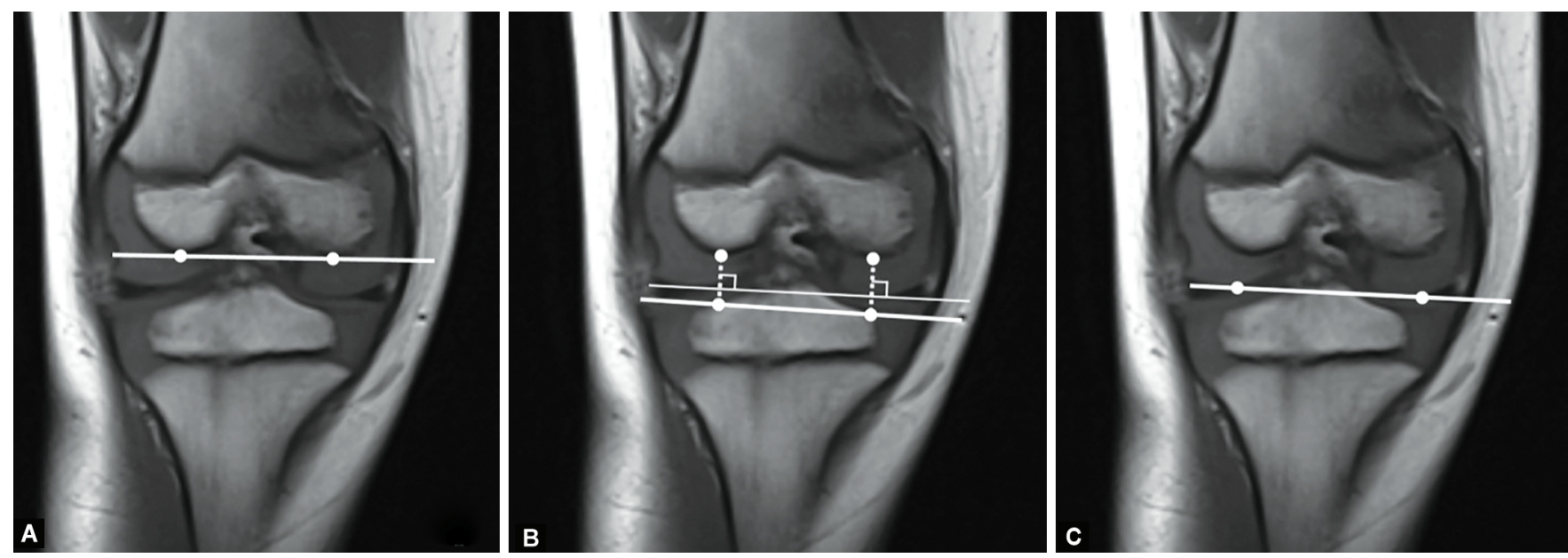

Figs $2 \mathrm{~A}$ to C: MRI images demonstrating the end bone and end cartilage orientation lines: (A) The convexities of the medial and lateral distal femoral condyles are used to draw the distal femur end bone line; (B) The points used for the distal femur are extended distally using an imaginary line orthogonal to the joint line [shown in (C)], and the points where they intersect the tibia are used to draw the proximal tibia end bone line; (C) The joint line is defined by the most peripheral point where the cartilage of the distal femur and proximal tibia meet in the medial and lateral joint spaces 
joint line and marked where these lines intersected the proximal tibial bone (Fig. 2B). A line connecting these two points represented PT. The joint line was drawn by connecting the most medial point at which the cartilage of the medial condyle of the femur articulated with the cartilage of the tibial plateau and the most lateral point at which the cartilage of the lateral condyle of the femur articulated with the cartilage of the tibial plateau (Fig. 2C).

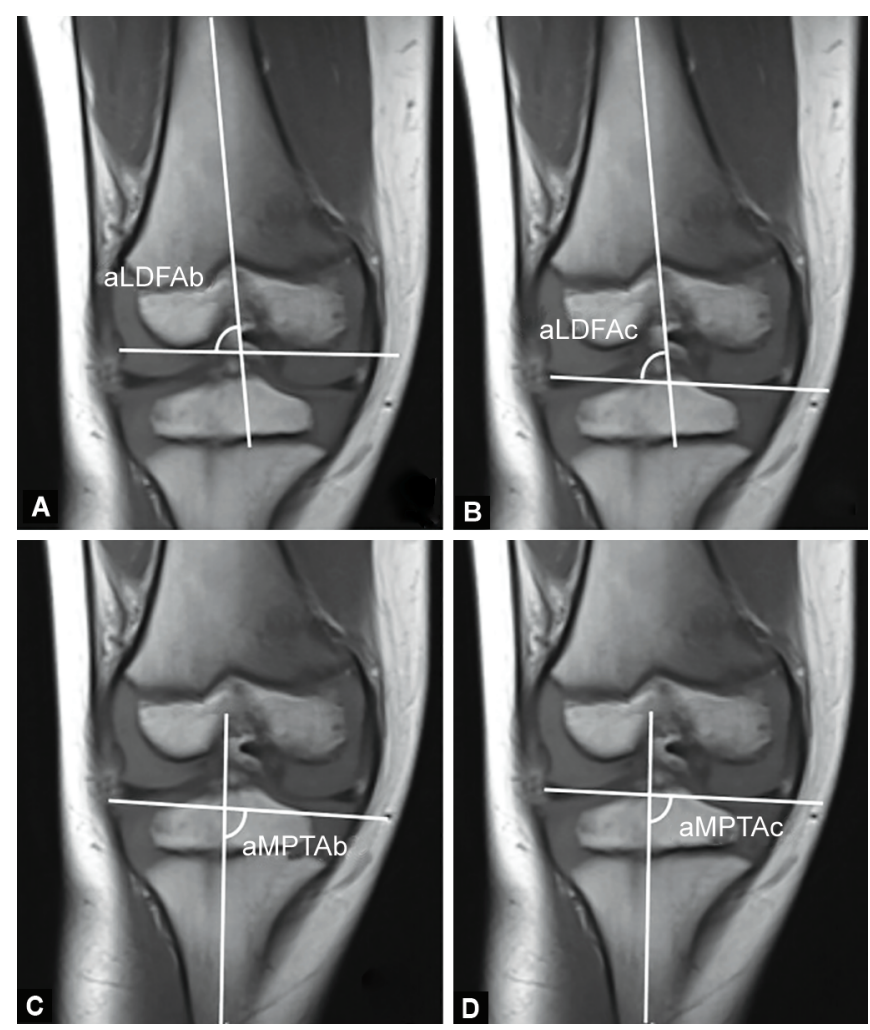

Figs $3 \mathrm{~A}$ to $\mathrm{D}$ : The lateral-sided angles between the anatomic axes and the corresponding end bone and end cartilage lines are used to define the (A) anatomic lateral distal femur angle of bone (aLDFAb) and (B) anatomic lateral distal femur angle of cartilage (aLDFAc). The medial sided angles are used to define the (C) medial proximal tibial angle of bone (MPTAb) and (D) medial proximal tibial angle of cartilage (MPTAC)

\section{Angle Measurements}

Four angles were measured using the angle tool in the PACS system. These were defined as the anatomical lateral distal femoral angle of bone (aLDFAb), the anatomical lateral distal femoral angle of cartilage (aLDFAc), the medial proximal tibial angle of bone (MPTAb) and the medial proximal tibial angle of cartilage (MPTAC) (Fig. 3).

\section{Physeal Orientation}

We considered measuring the orientation of the physis as a potential surrogate for the joint line as this would offer a simple practical alternative without the need for an arthrogram or an MRI clinically. We performed a pilot study in 20 specimens and did find excellent inter-relator reliability in measuring the orientation of the physis, with ICC $=0.81$. However, when we compared the orientation of the physis vs the orientation of the joint line we had a poor correlation with ICC $=0.13$, suggesting that the physis is not a reliable marker for joint orientation. Given this, we did not pursue the use of physealbased angles in this study.

\section{Statistical Analysis}

Absolute differences between aLDFA and MPTA of bone (aLDFAb, MPTAb) and cartilage (aLDFAc, MPTAc) were recorded. Paired twotailed $t$-tests were used to compare aLDFA and MPTA absolute differences of bone vs cartilage for the entire cohort, for males and females separately and for the subset of patients aged 4-6 years selected based on our analysis of the graphed data. An alpha cutoff of 0.05 was used for significance.

\section{Results}

We reviewed $133 \mathrm{MRI}$ studies from 2007 to 2017 and found 116 were deemed adequate for measurements. Of these, 56\% (65/116) were male with a mean of 8.9 (range 5-10) studies per year of age.

Average absolute differences between bone and cartilage angles for each measurement were recorded and are shown in Table 1. Interclass correlation coefficients for all four angles were excellent ${ }^{10}$ : aLDFAb (0.81), aLDFAc (0.76), MPTAb (0.77) and MPTAc (0.82). Intraclass coefficients were also excellent ${ }^{10}$ : aLDFAb (0.90), aLDFAc (0.92), MPTAb (0.89) and MPTAC (0.93). The mean difference in bone vs cartilage aLDFA was 2.1 at age 4 and 0.8 or lower at all other ages. The mean difference in bone vs cartilage MPTA was 1.4

Table 1: Number of studies per age range including averages of age and absolute values of the difference between aLDFA and MPTA angles measured at the bone and cartilage lengths, respectively, as well as ranges. Note the range in values of the younger age groupings

\begin{tabular}{lclllll}
\hline Age group & $N$ & Avg Age & $|a L D F A(b-c)|$ Avg & Range & & $\mid$ MPTA(b-c)|Avg \\
\hline 16 & 10 & 16.7 & 0.5 & $0-1.2$ & 0.6 & $0-1.6$ \\
15 & 10 & 15.5 & 0.3 & $0.1-0.5$ & 0.8 & $0.1-2.5$ \\
14 & 10 & 14.4 & 0.6 & $0-1.9$ & 0.5 & $0.2-1$ \\
13 & 10 & 13.5 & 0.6 & $0-2$ & 0.4 & $0-1.1$ \\
12 & 9 & 12.6 & 0.5 & $0-1.2$ & 0.6 & $0-1.4$ \\
11 & 10 & 11.4 & 0.6 & $0-1.4$ & 0.6 & $0-1.2$ \\
10 & 10 & 10.4 & 0.6 & $0.2-1.1$ & 0.6 & $0.1-1.1$ \\
9 & 10 & 9.7 & 0.7 & $0.2-1.2$ & 0.7 & $0.3-1.4$ \\
8 & 10 & 8.7 & 0.5 & $0-1.6$ & 0.9 & $0.1-2.7$ \\
7 & 8 & 7.6 & 0.8 & $0.2-1.4$ & 0.9 & $0.1-1.7$ \\
6 & 7 & 6.5 & 0.5 & $0-1.4$ & 0.9 & $0-2.4$ \\
5 & 5 & 5.5 & 0.6 & $0-1.9$ & 0.7 & $0.5-1.3$ \\
4
\end{tabular}

aLDFA, anatomic lateral distal femur angle of bone; MPTA, medial proximal tibial angle 
at age 4 , and 0.9 or lower at all other ages. There were no significant differences between aLDFA and MPTA when measured at the bone vs cartilage surfaces ( $p=0.42$ and $p=0.53$, respectively) while comparing the entire cohort. However, when the 4- to 6-year subgroup was compared there was a significant difference between bony and cartilaginous aLDFA ( $p=0.02$ ) but not MPTA ( $p$ $=0.88$ ). Figure 4 shows the graph of angle differences that shows a tendency for cartilage to be in relative valgus compared to bony measurements.

When separated by gender, both male and female subgroups aged 4-16 years did not show significant differences between aLDFAb and aLDFAc ( $p=0.47$ and $p=0.70$, respectively) or MPTAb and MPTAc ( $p=0.88$ and $p=0.16$, respectively). The difference in aLDFAb and aLDFAc in the male subgroup aged 4 to 6 years

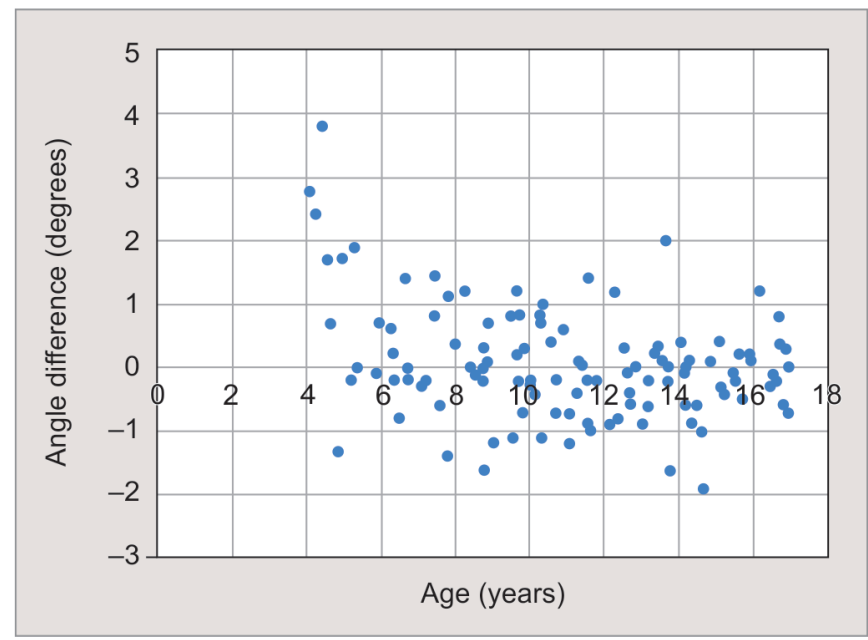

Fig. 4: Scatterplot showing the difference between cartilage values and bone values graphed against age. A positive value indicates that the cartilage measurement is in more valgus relative to the bony measurement. This plot helps show that in young patients the true distal femur measurement may be in more valgus than its bony representation. At approximately 6 years of age, this is less apparent and differences in values appear to be randomly distributed about zero remained ( $p=0.04$ ) but was not significant in the female subgroup $(p=0.28)$. There remained no significant difference between MPTAb and MPTAc for both 4- to 6-year-old males and females ( $p=0.97$ and $p=0.65$, respectively).

\section{Discussion}

Since the landmark papers of Paley and Tetsworth ${ }^{4,5}$ on limb deformity correction, there has been increased research in normative values for joint angles in younger patients. ${ }^{2,3,7}$ However, in younger patients, particularly those under the age of 6 , a large portion of the epiphysis is unossified cartilage. The current study demonstrates that bony measurements may not be accurate representations of the true joint angles in patients under 6 years old and may misrepresent the degree of valgus at the DF. Consideration for preoperative MRI or an intraoperative arthrogram is warranted in cases where a few degrees of difference would change the surgical approach. Although one might argue that this study found relatively small average differences, the ranges in younger age groups were large enough to be clinically important. For example, in the 4-year-old age group, the difference reached as high as $3.8^{\circ}$ and $4.2^{\circ}$ (aLDFA and MPTA), despite a more modest average difference (2.1 $1^{\circ}$ and $1.4^{\circ}$, respectively). As such, we favour the continued use of intraoperative arthrograms in these younger patients if mild changes in angulation may alter surgical management (Fig. 5). The senior author favours intraoperative arthrograms over MRIs as the latter would require additional sedation in the youngest age groups, whereas intraoperative arthrograms would be done under the same anesthesia as the planned operation.

Interestingly, when separated by gender, the difference between aLDFAb and aLDFAc for patients aged 4-6 years was only significant for males and not females. We propose two possible explanations for this finding. First, there were a small number of female patients in the 4-6-year-old age groupings and thus there could be inadequate powering to detect a significant difference. Alternatively, this difference could be explained by the earlier ossification of bone in females vs males. ${ }^{11}$

This study does have limitations. First, an MRI of the knee does not provide enough landmarks to make full-length measurements
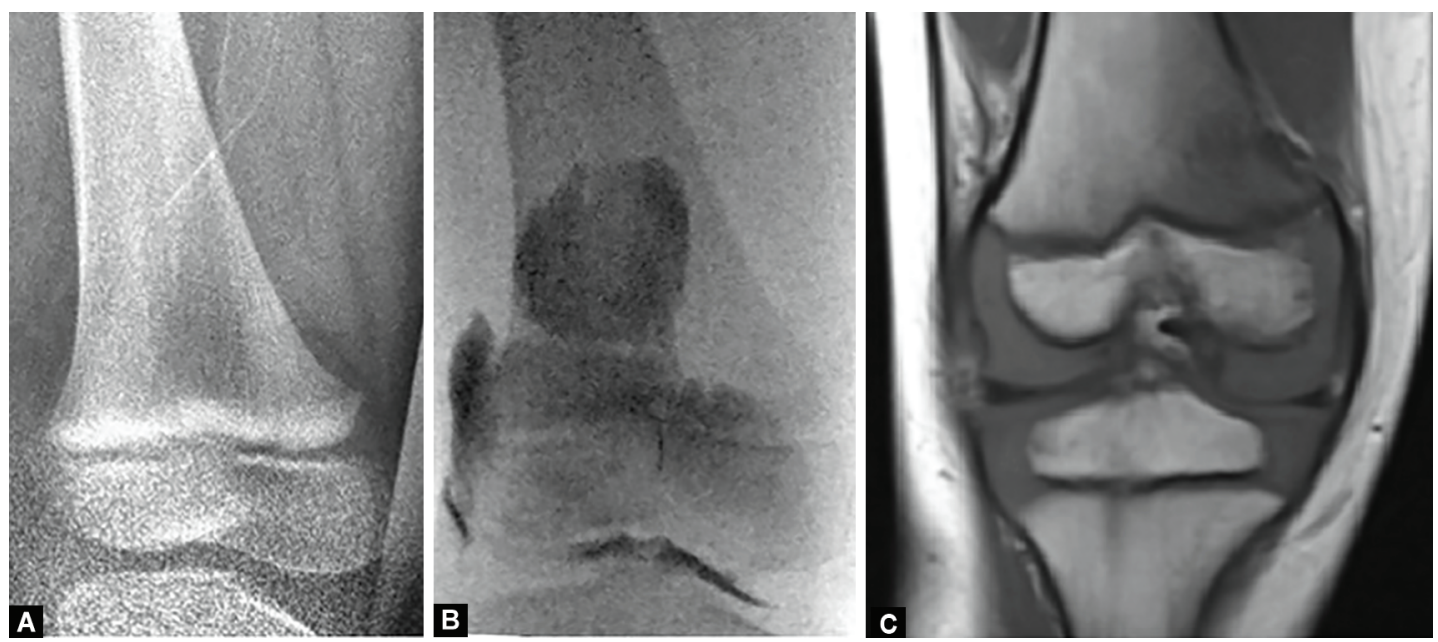

Figs $5 \mathrm{~A}$ to C: Comparison of a preoperative X-ray (A) to an intraoperative arthrogram (B) of the same patient for joint correction surgery. Note the difference in end-bone vs end-cartilage orientations shown on the arthrogram that are not appreciable on plain film X-ray, representing more valgus in the cartilage than the bone; (C) An MRI of a different patient (of similar bone age) demonstrates the asymmetrical thickness of cartilage with the medial side wider 
typically used in joint deformity correction, including the mechanical axis of the femur, and does not picture the entire bone for the ideal method of drawing anatomical axes. However, since all angle measurements were made using the same anatomical axis, the difference between aLDFAb and aLDFAc would not be affected by the misrepresentations of the anatomical axis. MRIs are also taken with the patient in the supine position. This is in contrast to the weight-bearing AP full-length standing radiographs where these angle measurements are described originally. Furthermore, this may introduce a rotational error. There is some evidence to suggest that in patients with small deviations from normal joint measurements, supine fluoroscopy measurements using an electrocautery cord are accurate enough representations of standing AP full-length radiographs. ${ }^{12}$ In regards to the rotation, Jamali et al. ${ }^{13}$ noted that LDFA measurements were not affected by the rotation of up to $12^{\circ}$ internal or external, and MPTA was only moderately affected with a large rotational element, suggesting the measurements reported are valid.

Importantly, this was a study of normal patients. Many children treated with limb deformity correction at younger ages have significant underlying pathology and thus the application of this data is limited. However, we felt it necessary to use normal patients to obtain enough MRI scans to understand the relationship with increasing age.

\section{Conclusion}

This anatomical study suggests that in patients age 6 years and younger, particularly males, it may be beneficial to pursue additional imaging for accurate joint angle measurements before joint corrective surgery as plain film radiographs may not accurately represent the true joint line.

\section{Clinical Significance}

This study supports the need for advanced imaging before joint corrective surgery or intraoperative arthrogram in patients under
6 years of age, particularly in cases that small angles about the knee may be of clinical importance.

\section{References}

1. Paley D. Principles of Deformity Correction. Berlin, Germany: Springer; 2002.

2. Popkov $D$, Lascombes $P$, Berte $N$, et al. The normal radiological anteroposterior alignment of the lower limb in children. Skeletal Radiol 2015;44(2):197-206. DOI: 10.1007/s00256-014-1953-z.

3. Cooke TD, Li J, Scudamore RA. Radiographic assessment of bony contributions to knee deformity. Orthop Clin North Am 1994;25(3):387-393.

4. Paley D, Tetsworth K. Mechanical axis deviation of the lower limbs. preoperative planning of multiapical frontal plane angular and bowing deformities of the femur and tibia. Clin Orthop Relat Res 1992;280:65-71.

5. Paley D, Tetsworth K. Mechanical axis deviation of the lower limbs. preoperative planning of uniapical angular deformities of the tibia or femur. Clin Orthop Relat Res 1992(280):48-64.

6. Salenius P, Vankka E. The development of the tibiofemoral angle in children. J Bone Joint Surg Am 1975;57(2):259-261. DOI: 10.2106/00004623-197557020-00021.

7. Sabharwal S, Zhao C, Edgar M. Lower limb alignment in children: reference values based on a full-length standing radiograph. J Pediat Orthop 2008;28(7):740-746. DOI: 10.1097/BPO.0b013e318186eb79.

8. Gordon JE, Chen RC, Dobbs MB, et al. Interobserver and intraobserver reliability in the evaluation of mechanical axis deviation. J Pediatr Orthop 2009;29(3):281-284. DOI: 10.1097/BPO.0b013e31819b9188.

9. Ferguson DO, Fernandes JA. Lower limb alignment. Orthopaedics and Trauma 2016;30(6):539-546. DOI: 10.1016/j.mporth.2016.09.002.

10. Fleiss JL. The design and analysis of clinical experiments. New York: Wiley; 1986.

11. Pryor JW. Difference in the ossification of the male and female skeleton. J Anat 1928;62(Pt 4):499-506.

12. Sabharwal $S$, Zhao C. Assessment of lower limb alignment: supine fluoroscopy compared with a standing full-length radiograph. J Bone Joint Surg Am 2008;90(1):43-51. DOI: 10.2106/JBJS.F.01514.

13. Jamali AA, Meehan JP, Moroski NM, et al. Do small changes in rotation affect measurements of lower extremity limb alignment? J Orthop Surg Res 2017;12(1):77. DOI: 10.1186/s13018-017-0571-6. 